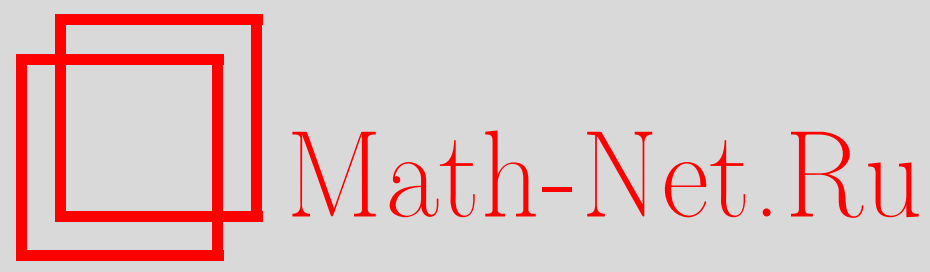

С. В. Людковский, Измеримость автоморфизмов топологических групп, Матем. заметки, 2000, том 68, выпуск 1, 105-112

DOI: https://doi.org/10.4213/mzm924

Использование Общероссийского математического портала Math-Net.Ru подразумевает, что вы прочитали и согласны с пользовательским соглашением http://www.mathnet.ru/rus/agreement

Параметры загрузки:

IP: 18.208 .226 .222

26 апреля 2023 г., $17: 46: 26$

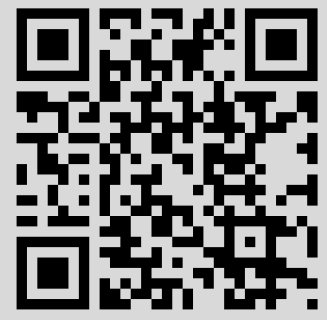




\section{ИЗМЕРИМОСТЬ АВТОМОРФИЗМОВ ТОПОЛОГИЧЕСКИХ ГРУПП}

\section{С. В. Людковский}

В статье исследована проблема о соотношениях между измеримостью и непрерьвностью алгебраических автоморфизмов топологических групп в зависимости от типа групп. Разобраны случаи и доказаны соответствующие теоремы, когда измеримость автоморфизма влечет за собой его непрерывность, например, для сепарабельной локально компактной группы и для автоморфизма, измеримого относительно неотрицательной меры Хаара. С другой стороны, доказано существование и приводятся примеры неметризуемых несепарабельных компактных групп с мерами Хаара и не локально компактных сепарабельных метризуемых групп с квазиинвариантными мерами $\mu$ относительно плотных подгрупп, для которых существуют $\mu$-измеримые разрьвные автоморфизмы.

Библиографоия: 18 названий.

В работе [1] было исследовано существование разрывных представлений локально компактных групп. Данная работа посвящена исследованию проблемы о соотношениях между измеримостью и непрерьвностью автоморфизмов топологических групा $G$ в зависимости от типа групп. Очевидно, что семейство всех алгебраических автоморфизмов $g$ группы $G$ является группой, которая обозначается $\operatorname{Aut}(G)$, а автоморфизмы характеризуются тем, что $g(a b)=g(a) g(b)$ для любых $a, b \in G$. Здесь рассматриваются $T_{0}$-группы, которые также являются тихоновскими пространствами согласно теореме 8.4 из [2].

1. Определения. Автоморфизм $g$ назьвается $\mu$-измеримым, если $g^{-1}(A) \in A f(G$, $\mu$ ) для любых $A \in B f(G)$, где $\mu$ обозначает $\sigma$-аддитивную неотрицательную меру, определенную на $\sigma$-алгебре $A f(G, \mu)$, которая является пополнением борелевской $\sigma$-алгебры $B f(G)$ топологической групшы $G$ с помощюю подмножеств $E \subset F$, где $F \in B f(G)$ и $\mu(F)=0$. Мера $\mu$ назьвается левоинвариантной мерой Хаара, если $\mu(b A)=\mu(A)$ для любых $A \in A f(G, \mu)$ и $b \in G$. Если $H$ является подгруппой в $G$ и $\mu^{h}$ эквивалентна $\mu$ для любых $h \in H$, то мера $\mu$ на $G$ назьвается квазиинвариантной относительно подгруппы $H$ в $G$, где $\mu^{h}(A):=\mu\left(h^{-1} A\right)$ для любых $\mu$-измеримых подмножеств $A \subset G$ и элементов $h \in H$, a $h^{-1} A:=\left\{a: a=h^{-1} b, b \in A\right\}$ и $H \subset G ; h^{-1}$ обозначает обратный элемент для $h$. Как обычно, $A B:=\{c: c=a b, a \in A, b \in B\}$ для подмножеств $A$ и $B$ в $G$.

Для удобства записи далее используется обозначение $\int_{G} f(x) \mu(d x)$ вместо $\int_{G} f(x) \times$ $d \mu(x)$. Как обычно, $L^{p}(G, \mu, \mathbb{C})$ обозначает пространство классов эквивалентности измеримых комплекснозначных функций $f: G \rightarrow \mathbb{C}$, имеющих конечную норму

$$
\|f\|_{p}:=\left(\int_{G}|f(x)|^{p} \mu(d x)\right)^{1 / p}
$$


где $1 \leqslant p<\infty$, а $\mu$ - неотрицательная мера.

В топологическом пространстве $X$ подмножество $A \subset X$ назьвается множсеством первой категории, если $A$ является объединением $A=\bigcup_{i} A_{i}$ счетного семейства нигде не плотных множеств $A_{i}$. Пусть $J$ обозначает семейство всех множеств первой категории в $X$. Множество $A$ называется нетощим, если $A \notin J$. Множество $A$ назьвается открытым по модулю $J$, если существует открытое множество $Y$ такое, что $A \backslash Y$ и $Y \backslash A$ являются множествами первой категории. Свойство открытости множества $A$ по $\bmod J$ называется свойством Бәра, семейство таких множеств обозначается через $\mathbf{B}$. Говорят, что отображение $f: X \rightarrow H$ в топологическоепространство $H$ обладает свойством Бәра, если $f^{-1}(F) \in \mathbf{B}$ для любых замкнутых подмножеств $F$ в $H$.

2. Теорема 1. Пусть на локально компактной сепарабельной группе $G$ заданы левоинвариантная мера Хаара $\mu$ и $\mu$-измеримый автоморфизм $g \in \operatorname{Aut}(G)$. Тогда $g$ непрерывно.

Теорема 2. Пусть $G_{i}$ - полные сепарабельные топологические группы с равномерностями $\tau_{i}$ при $i=1,2,3$, с левоквазиинвариантными вероятностными мерами $\mu_{i}\left(\right.$ на $G_{i}$ относительно $G_{i+1}$ при $\left.i=1,2\right)$ и непрерывными факторами квазиинвариантности $d_{\mu_{i}}(h, g):=\mu_{i}^{h}(d g) / \mu_{i}(d g)$ на $G_{i+1} \times G_{i}$ nри $i=1,2$, где $h \in G_{i+1} \subset G_{i}, g \in G_{i}$. Если $g \in \operatorname{Aut}\left(G_{1} \times G_{2}\right)$ и ограничения $\left.g\right|_{G_{i}} \mu_{i}$-измеримы при $i=1,2$, то $\left.g\right|_{\left(G_{2}, \tau_{2}\right)}$ непрерывно.

ТЕорема 3. Пусть $G$ - сепарабельная нетощая топологическая группа и автоморфизм g обладает свойством Бәра. Тогда $g$ непрерывно на $G$.

ДоКАЗАТЕЛЬСТВО ТЕОРЕмЫ 1 . В силу теоремы 8.3 из [2] $T_{0}$-групша метризуема тогда и только тогда, ког да она имеет счетную открытую базу в $e$, причем метрика может быть выбрана левоинвариантной; следовательно, группа $G$ метризуема (также в случае теоремы 2 для $G_{i}$ ). В силу следствия 3.3 .11 из [3] хаусдорфово пространство локально компактно в том и только том случае, если оно гомеоморфно открытому подпространству некоторого компакта. Согласно теореме Кантора (см. [3, теорема 4.3.8]) метрическое пространство $(G, \rho)$ полно тогда и только тогда, когда каждая убьвающая последовательность $F_{1} \supset F_{2} \supset \cdots$ непустых замкнутых подмножеств пространства $G$ таких, что

$$
\lim _{i \rightarrow \infty} \delta\left(F_{i}\right)=0
$$

имеет непустое пересечение: $\bigcap_{i=1}^{\infty} F_{i} \neq \varnothing$, где $\rho$-метрика на $G$,

$$
\delta(F):=\sup _{a, b \in F} \rho(a, b) .
$$

Отсюда вытекает, что $G$ - полное метрическое пространство. В силу определения $\mu$-измеримости из п. $1 g^{-1}(A) \in A f(G, \mu)$ для любых $A \in B f(G)$. В силу теоремы Лузина (см. [4, теорема 2.3.5]) для любого $b>0$ существует компактное подмножество $C_{b} \subset G$ такое, что $\mu\left(G \backslash C_{b}\right)<b$ и $\left.g\right|_{C_{b}}$ непрерывно. Пусть $b<\mu(G \cap V)$ для некоторой (открытой) окрестности $V \ni e$ в $G$. В силу следствия III.12.4 из [5] для любых двух $\mu$-измеримых подмножеств $A$ и $B$ в $G$, каждое из которых не является локально $\mu$-нулевым, $A B$ имеет внутреннюю точку, причем $е$ является внутренней точкой в $A A^{-1}$. Тогда $C_{b} C_{b}^{-1}$ - это окрестность единичного элемента $e$ в $G$. Но $\left.g\right|_{C_{b} C_{b}^{-1}}$ непрерывно, так как $g(a c)=g(a) g(c)$ и $(g(a))^{-1}=g\left(a^{-1}\right)$ для любых $a, c \in G$. В силу предложения III.1.14 из [5] подгруппа $H$, открытая в $G$, является также замкнутой в $G$. С другой 
стороны, подгруппа $H$ в $G$ открыта тогда и только тогда, когда внутренность $H$ непуста (см. [5, упражнение III.10]). Напомним, что топологическая групша $H$ называется компактно порожденной, если существует компактное подмножество $C$ в $H$ такое, что подгруппа $H_{C}$, порожденная $C$, совпадает с $H$. Группа $H$ компактно порождена тогда и только тогда, когда существует открытое подмножество $U$ в $H$, замыкание котрого $\bar{U}$ компактно и $U$ порождает $H$ (см. [5, упражнение III.35]). Для локально компактной группы $G$ следуюшие утверждения эквивалентны:

а) $G$ является $\sigma$-компактной;

б) $G$ является линделёфовьм пространством;

в) для любой замкнутой подгрупшы $H$ в $G$ пространства $H$ и $G / H$ являются $\sigma$-компактными.

Поэтому компактно порожденная подмножеством $C$ подгруппа $G_{C}$ открыто-замкнута в $G$. Алгебраический гомоморфизм $f: G \rightarrow K$ топологических групп $G$ и $K$ непрерьвен в $e \in G$ тогда и только тогда, когда $f$ непрерывен на $G$ (см. [5, §III.2.7]); следовательно, $g$ непрерьвен на $G$, так как он непрерьвен на $G_{C}$. При этом $g\left(G_{C}\right)$ также компактно порождена, так как $g(C)$ компактно согласно теореме 3.1.10 из [3].

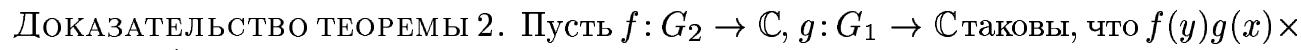
$d_{\mu_{1}}(y, x) \in L^{1}\left(G_{2} \times G_{1}, \mu_{2} \times \mu_{1}, \mathbb{C}\right)$. Напомним, что измеримое пространство $(X, V)$ назьвается радоновым, если оно содержит компактный подкласс $K$, аппроксимирующий снизу каждую неотрицательную меру на $(X, V)$, где $X$ - множество с $\sigma$-алгеброй $V$ его подмножеств, $K \subset V$, а класс подмножеств $K$ назьвается компактным, если для любой последовательности $\left\{K_{n}: n \in \mathbb{N}\right\}$ в $K$ из $\bigcap_{n=1}^{\infty} K_{n}=\varnothing$ следует, что существует $m \in \mathbb{N}$, для которого $\bigcap_{n=1}^{m} K_{n}=\varnothing$. В силу теоремы I.1.4 из [6] сепарабельное полное метрическое пространство $X$ является топологическим радоновым пространством $(X, B f(X))$. Отсюда следует, что меры $\mu_{i}$ на $G_{i}$ радоновы. В силу теоремы Фубини (см. [5, теорема II.9.8] или $[4, \S 2.6 .2]$ )

$$
\begin{aligned}
\int_{G} \int_{G^{\prime}} f(y) g(z) d_{\mu_{1}}\left(y^{-1}, z\right) \mu_{2}(d y) \mu_{1}(d z) & =\int_{G^{\prime}} \int_{G} f(y) g\left(y^{-1} x\right) \mu_{2}(d y) \mu_{1}(d x) \\
& =\int_{G}(f * g)(x) \mu_{1}(d x)
\end{aligned}
$$

следовательно, $\mu_{1}$-почти всюду существует свертка $f * g$ на $G$, т.е. $f * g \in L^{1}\left(G, \mu_{1}, \mathbb{C}\right)$. Беря характеристические функции $\chi_{A} \quad \mu_{1}$-измеримых подмножеств $A$ в $G_{1}$, мы получим, что для любого $b>0$ существует компактное подмножество $C_{b}$ в $G_{2}$ такое, что $\mu_{2}\left(G_{2} \backslash C_{b}\right)<b$ и $\left.\mu_{1}(A \cap x B)\right|_{C_{b}}$ непрерывно по $x \in C_{b}$, где $A, B \in A f\left(G, \mu_{1}\right)$. Это объясняется тем, что для $f(y)=\chi_{A}(y)$ и $g(x)=\chi_{B}(x)$ имеется неравенство

$$
\int_{G} \int_{G^{\prime}} \chi_{A}(y) \chi_{B}\left(y^{-1} x\right) \mu_{2}(d y) \mu_{1}(d x) \leqslant \int_{G} \int_{G^{\prime}} \chi_{A}(y) \chi_{G}(z) d_{\mu_{1}}\left(y^{-1}, z\right) \mu_{2}(d y) \mu_{1}(d z) .
$$

Поэтому подгруппа $G_{C_{b}}$, компактно порожденная в $G_{1}$ подмножеством $C_{b}$, является $G_{\delta}$-подмножеством в $G_{1}$. Итак, оно удовлетворяет теореме Бэра о категории (см. [3, теорема 3.9.3]): в полном по Чеху пространстве $X$ объединение $A=\bigcup_{i=1}^{\infty} A_{i}$ любой последовательности нигде не плотных множеств является коплотным множеством, т.е. его дополнение $X \backslash A$ всюду плотно в $X$, где тихоновское пространство $X$ называется $n$ олным по Чеху, если существует компактификация $c X$ пространства $X$ такая, что нарост 
$c X \backslash c(X)$ является $F_{\sigma}$-множеством в $X$, т.е. счетным объединением замкнутых множеств (его дополнение является $G_{\delta}$-множеством). В самом деле, согласно теореме 3.9 .6 из [3]: полнота по Чеху наследуется замкнутыми множествами и $G_{\delta}$-множествами. Тогда $\left.g\right|_{C_{b} C_{b}^{-1}}$ непрерывно и, следовательно, $g$ непрерьвно на $G_{2}$.

ДОКАЗАТЕЛЬСТВО ТЕОРЕМЫ 3. Поскольку $g$ обладает свойством Бэра, это эквиваленто тому, что $g$ является поточечным пределом последовательности непрерьвных отображений $g_{n}, n \in \mathbb{N}$; следовательно, и $g^{-1}$ обладает свойством Бэра (см. [7, 32$]$ ). Тогда существует почти открытое подмножество $A$ в $G$ такое, что ограничение $\left.g\right|_{A}$ непрерьвно, так как $G$ нетощая. Отображение $\operatorname{inv}(b):=b^{-1}$ для любых $b \in G$ непрерывно; следовательно, $A^{-1}=\operatorname{inv}(A)$ тоже почти открыто, так как inv является гомеоморфизмом группы $G$. С другой стороны, $\left.g\right|_{A^{-1}}$ непрерывно, так как $g\left(a^{-1}\right)=(g(a))^{-1}$ для любых $a \in A$. Отображение $L_{b}: G \rightarrow G$ непрерывно, где $L_{b} a:=b a$ для любых $a, b \in G$. Тогда можно выбрать $A$ так, чтобы $e \in A$, где $e$ обозначает единичный элемент груп-

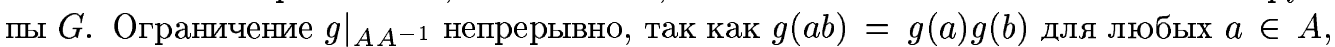
$b \in A^{-1}$, а отображение умножения $G \times G \ni(a, b) \mapsto a b \in G$ непрерьвно. Согласно теореме Банаха-Куратовского-Петтиса, если множество $E$ содержит нехудоепочти открытое подмножество топологической групшы $G$, то $E E^{-1}$ является окрестностью единичного элемента (см. [8, гл. $6, \S \mathrm{P}\left(\right.$ в)]); следовательно, $A A^{-1}$ является окрестностью $e$ в $G$. Непрерьвность $g$ на открытом подмножестве в $G$ эквивалентна непрерьвности $g$ на всей группе $G$.

3. ОПРЕДЕЛЕНИЕ. Пусть $G$ - топологическая группа с вероятностной мерой $\mu$ и левоинвариантной метрикой $\rho$ на $G$. Обозначим через $\operatorname{Aut}_{\mu}(G)$ подгруппу в $\operatorname{Aut}(G), \operatorname{coc}$ соящую из $g \in \operatorname{Aut}(G)$ таких, что $g(A f(G, \mu)) \subset A f(G, \mu)$ и $g^{-1}(A f(G, \mu)) \subset A f(G, \mu)$.

Очевидно, что имеется левоинвариантная псевдометрика (или метрика на классах эквивалентности), задаваемая формулами

$$
\bar{\rho}(g, h):=\bar{\rho}\left(h^{-1} g, \mathrm{id}\right), \quad \bar{\rho}(f, \mathrm{id}):=\int_{G} \rho(f(x), x) \mu(d x) .
$$

\section{Tеорема. Существуют}

1) неметризуемые компактные группы с мерами Хаара $\mu$,

2) не локально компактные сепарабельные метризуемые полной метрикой группы $G$ с квазиинвариантными мерами $\mu$ относительно плотных подəpynn $G^{\prime}$

такие, что они имеют $\mu$-измеримые разрывные автоморфизмы.

ДокАЗАтЕЛЬСтво. 1) Рассмотрим компактную группу $G=\mathbf{T}^{\mathbf{c}}$ в тихоновской топологии декартова (прямого) произведения топологических групп (см. $[3, \S 8.5 .4]$ и $[2, \S 6])$, где $\mathbf{T}=\mathbb{R} / \mathbb{Z}-$ тор, с $:=\operatorname{card}(\mathbb{R})$. Тогда мера Хаара $\mu$ на группе $G$ является произведением вероятностных мер Хаара $\nu_{i}$ на каждой групе $\mathbf{T}_{i}$, где $\mathbf{T}_{i}=T$ для любых $i \in \mathbb{R}$. Пусть $C$ является компактньм подмножеством в $\mathbf{T}_{i}$ с $\nu_{i}(C)=0$. Тогда компактно порожденная подгруппа $G_{C}$ является $\sigma$-компактной и $G_{C} \in B f\left(\mathbf{T}_{i}\right)$. Поскольку $\nu_{i}(x)=0$ для любой точки $x \in \mathbf{T}_{i}$, никакое счетное семейство таких $G_{C_{j}}$ не может покрыть всей группы $\mathbf{T}_{i}$.

Например, в качестве $C$ можно взять подмножество, гомеоморфное Канторовому множеству $D^{\aleph_{0}}$ (см. $\left.[3, \S 2.3]\right)$, где $D=\{0,1\}$ обозначает двухточечное дискретное пространство. Используя их сдвиги, можно по индукции построить бесконечные семейства 
таких подгрупп $G_{C}$ с попарньми пересечениями, равньми $e$. Множество $S$ всех таких семейств частично упорядочено по включению. По лемме Куратовского-Цорна [3] каждое линейно упорядоченное подмножество $P$ имеет максимальньй элемент $\bigcup_{p \in P} p$ в $S$. Поэтому для любого $i$ имеются семейства $\left\{G_{C_{j}}:=G_{i, j}: j \in \mathbb{R}\right\}$ с $\bigcup_{j} G_{i, j}=\mathbf{T}_{i}$.

С другой стороны, $\nu_{i}\left(G_{C}\right)=0$, так как

$$
\lambda(A B)=\int_{H} \chi_{A}(x) \lambda(x B) \lambda(d x)=\int_{H} \chi_{A}(x)\left(\int_{H} \chi_{x B}(y) \lambda(d y)\right) \lambda(d x),
$$

где $\lambda$ - мера, эквивалентная левоинвариантной (неотрицательной) мере Хаара на локально компактной группе $H$ (в данном случае $H=\mathbf{T}$ ). Тогда равенство $\lambda(B)=0$ эквивалентно $\lambda_{x}(B)=0$ для любьх $x \in H$ и $B \in A f(H, \lambda)$; следовательно, $\lambda(A B)=0$ для любых подмножеств $A$ и $B$ в $A f(H, \lambda)$ с $|\lambda|(A)+|\lambda|(B)=0$, где $|\lambda|$ обозначает вариацию меры $\lambda$. Напомним, что для топологического пространства $X$, его покрытия $\left\{A_{s}\right\}_{s \in S}$ и согласованного семейства отображений $f_{s}: A_{s} \rightarrow Y$ в топологическое пространство $Y$, т.е.

$$
\left.f_{s_{1}}\right|_{A_{s_{1}} \cap A_{s_{2}}}=\left.f_{s_{2}}\right|_{A_{s_{1}} \cap A_{s_{2}}}
$$

для любых $s_{1}, s_{2} \in S$, можно определить отображение $f: X \rightarrow Y$, полагая $f(x)=f_{s}(x)$ для любых $x \in A_{s}$, которое называется комбиначией отображсений $\left\{f_{s}\right\}_{s \in S}$ и обозначается $\nabla_{s \in S} f_{s}$ (см. $\left.[3, \S 2.1]\right)$.

Тогда имеются автоморфизмы $f: G_{0} \rightarrow G_{0}$, удовлетворяющие следующим условияM:

i) $f_{i, j}:=\left.f\right|_{G_{i, j} \otimes \bigotimes_{k \neq i} e_{k}}-$ алгебраические изоморфизмы групп $G_{i, j} \otimes \bigotimes_{k \neq i} e_{k}$ с соответствуюшими группами $G_{\left(i^{\prime}, j^{\prime}\right)} \otimes \bigotimes_{k \neq i^{\prime}} e_{k}$, где $e_{k}$ обозначает единичньй элемент в $\mathbf{T}_{k}, \sigma$ - элемент симметрической группы $\Sigma\left(\alpha^{2}\right)$ всех биекций ординала $\alpha^{2}$ мощности с $:=\operatorname{card}(\mathbb{R}),\left(i^{\prime}, j^{\prime}\right):=\sigma(i, j)$;

ii) $\sigma$ таков, что $\operatorname{card}\left\{\sigma(i, j)=\left(i^{\prime}, j^{\prime}\right): i \neq i^{\prime}\right.$ и $j \neq j^{\prime}, G_{i, j}$ содержит элементы бесконечных порядков $\}=\beta \geqslant \aleph_{0}, \sigma$ не переставляет $i$ с $j ; \operatorname{card}\left\{j^{\prime}: \sigma(i, j)=\left(i^{\prime}, j^{\prime}\right)\right.$, $\left.j^{\prime} \neq j\right\} \leqslant \aleph_{0}$ для любого заданного $i \in \alpha$; комбинация семейства отображений $\left\{\pi_{i^{\prime}} \circ f_{i, j}: j\right\}$ дает некоторьй непрерьвньй автоморфизм для любого $\mathbf{T}_{i}$, где $\pi_{i}: G \rightarrow \mathbf{T}_{i}$ обозначают проекции;

iii) $G_{0}$ - подгруппа в $G$ такая, что $\pi_{i}\left(G_{0}\right)$ для любых $i$ и $j$ либо содержит в себе $G_{i, j}$, либо пересекается с ней по $e_{i}$;

iv) $G_{i, j}$ и $G_{\sigma(i, j)}$ - изоморфные топологические групшы для любых $i, j$ и $\sigma$ из условия ii).

Семейство $J$ всех таких автоморфизмов частично упорядочено поправилу: $A=\left(G_{0}^{1}, f^{1}\right)$ $<B=\left(G_{0}^{2}, f^{2}\right)$ и равносильно двум условиям $G_{0}^{1} \subset G_{0}^{2}$ и $\left.f^{2}\right|_{G_{0}^{1}}=f^{1}$.

В силу леммы Куратовского-Цорна имеются элементы в $J$, так что $\beta=$ с и $G_{0}=G$, так как в противном случае каждое вполне упорядоченное подмножество $J_{0}$ в $J$ имело бы $\max \left(J_{0}\right)$ или с $\beta<$ с или $G_{0} \neq G$. В последнем случае по индукции можно построить элемент $X=\left(G_{0}^{2}, f^{2}\right)>\max \left(J_{0}\right)$. Пусть $f$ является таким автоморфизмом группы $G_{0}=G$. Тогда он разрывен в силу условий i), ii), так как подгруппы в $\mathbf{T}$, порожденные элементами бесконечного порядка, плотны в Т. Если $U=\prod_{i} U_{i}$ является открытым подмножеством в $G$ и принадлежит базе $Q$ тихоновской топологии в $G$, то в силу условий ii)-iv) $f^{-1}(U) \in A f(G, \mu)$, так как $U_{i} \neq \mathbf{T}_{i}$ только для конечного семейства индексов $i$. 
Мера $\mu$ радонова и регулярна на $G$; следовательно, $\mu(\bar{U} \backslash U)=0$ для любого $U \in Q$, где $\bar{U}$ обозначает замькание $U$. Для любого открытого $V$ в $G$ и любого $c>0$ существует конечное покрытие подмножества $\bar{V}$ конечным семейством $U(s) \in Q, s=1, \ldots, n$, так что $\mu\left(\bigcup_{s=1}^{n} U(s) \backslash \bar{V}\right)<c$. Также сушествует последовательность $U^{\prime}(s) \in Q$ такая, что $U^{\prime}(s+1) \supset U^{\prime}(s)$ и

$$
\lim _{s \rightarrow \infty} \mu\left(V \backslash U^{\prime}(s)\right)=0 .
$$

Очевидно, что $\mu\left(f^{-1}(\bar{U} \backslash U)\right)=0$ для любого $U \in Q$; следовательно,

$$
\mu\left(f^{-1}\left(V \backslash \bigcup_{s \in \mathbb{N}} U_{s}^{\prime}\right)\right)=0
$$

поэтому $f^{-1}(V) \in A f(G, \mu)$. Для локально компактной группы $H$ любая лево(право)инвариантная неотрицательная мера $\lambda$ на $H$ является регулярной, т.е. для любого открытого подмножества $V \subset H$ вьполнено $\lambda(V)=\sup \{\lambda(F): F$ компактно и $F \subset V\}$ и для любого $A \in A f(H, \lambda)$ с $\lambda(A)<\infty$ выполнено $\lambda(A)=\inf \{\lambda(V): V$ открыто и $V \supset A\}$ (см. [4, теорема 2.7.14] и $[2, \S 11.22, \S 11.23, \S 15])$. Отсюда следует, что автоморфизм $f \mu$-измерим.

2) Пусть $G^{\prime}$ - плотная подгруппа в $G$, причем $G^{\prime}$ имеет семейство $K$ однопараметрических подгрупп мощности $\operatorname{card}(K)=\mathrm{c}\left(\mu\right.$ и $G^{\prime}$ можно так выбрать). Говорят, что для топологической групшы $H$ ее подпространство $X$ топологически порождает $H$, если в $H$ не существует замкнутой подгруппы, содержащей $X$. Пусть группа $F$ порождена множеством $X$. Тогда $F$ называется свободной әруппой со со свободным базисом $X$, если каждое соотношение

$$
\prod_{i=1}^{m} x_{i}^{\varepsilon_{i}}=1_{F}
$$

может быть приведено к $1_{F}$ последовательным сокращением пар вида $x^{\varepsilon} x^{-\varepsilon}$, где $x_{i} \in X$, $\varepsilon_{i} \in\{-1,1\}, m \in \mathbb{N}$, a $1_{F}$ - единичньй элемент в $F$. Единственная топологическая группа $F$ со свойствами $(\mathrm{F} 1)-(\mathrm{F} 3)$ назьвается свободной топологической группой, порожденной топологическим пространством $X$, где

(F1) $X$ - подпространство в $F$;

(F2) $X$ топологически порождает $F$;

(F3) для любого непрерывного отображения $\varphi: X \rightarrow G$ в топологическую групу $G$ существует непрерьвный гомоморфизм $\Phi: F \rightarrow G$ такой, что $\varphi(x)=\Phi(x)$ для любых $x \in X[9]$.

Каж дая топологическая группа является также равномерным пространством, и существует его пополнение (см. [3, $§ 8.1 .17$, теорема 8.3.12]), являющееся полной топологической группй, которая назьвается свободной полной топологической группой. Пусть $B$ - наименьшая замкнутая подгруппа в $F$, содержащая все элементы вида $a b a^{-1} b^{-1}$ для любых $a, b \in F$. Тогда факторгруппа $F / B$ назьвается свободной (полной) абелевой әруппой.

Предположим также, что существует окрестность $W \ni$ в в $G$ такая, что $K \cap W$ плотно в $W$. Например, для групшы Банаха-Ли над $\mathbf{L}=\mathbb{R}$ или неархимедовьп локальньпм полем $\mathbf{L}$ можно взять $K$ и задать прямое (декартово) произведение (см. [2, $§ 2.3]$ )

$$
\left(\prod_{j=1}^{\infty}{g_{j}}^{\mathbf{L}}\right) \times \prod_{Y \in K} Y
$$


где $g^{\mathbf{L}}$ - абстрактная свободная однопараметрическая топологическая абелева групша, порожденная элементом $g$. Таким образом, $g^{\mathrm{L}}$ равна $F / B_{1}$ с порождающим топологическим пространством $X=\left\{g^{a}:=(g, a): a \in \mathbf{L}\right\} \subset\{g\} \times \mathbf{L}$, причем $B_{1} \supset B$ и $B_{1}$ также содержит все элементы вида $g^{a+b} g^{-a} g^{-b}$, т.е. в $F / B_{1}$ выполняется тождество $g^{a+b}=g^{a} g^{b}$ для любых $a, b \in \mathbf{L}$ (в общем случае она может не иметь никакого отношения к однопараметрическим подгруппам в исходной групше $G$ ).

В случае группы диффеоморфизмов $G:=\operatorname{Diff}^{t}(M)$ класса гладкости $C^{t}$ с $1 \leqslant t \leqslant \infty$ соответствующего локально компактного многообразия $M$ над $\mathbf{L}:=\mathbb{R}$ или неархимедовым локальным полем $\mathbf{L}$ снабдим $\operatorname{Diff}^{t}(M)$ компактно открытой топологией [10]-[12]. Можно аналогично рассмотреть не локально компактные сепарабельные банаховы многообразия $M$ и снабдить сепарабельные подгруппы полных групп диффеоморфизмов соотвествуюшими топологиями (см. те же работы). Выберем

$$
W:=\left\{g \in G: \rho_{U}^{l}(g, \mathrm{id}):=\sum_{i, j \in E}\left\|\left(g_{i, j}-\mathrm{id}_{i, j}\right)\right\|_{C^{l}\left(U_{i, j}, Y\right)}<\varepsilon\right\},
$$

где $Y=\mathbf{L}^{m}=T_{x} M, g_{i, j}:=\varphi_{i} \circ g \circ \varphi_{j}^{-1}$ под знаком суммы заданы на $U_{i, j} \subset Y$ пересечении областей определения $g_{i, j}$ с $\varphi_{j}\left[U \cap\left(\bigcup_{s \in E} \varphi_{s}\left(U_{s}\right)\right)\right],\left(U_{j}, \varphi_{j}\right)$-карты атласа многообразия $M, U_{j}$ открыты в $M$, а $\varphi_{j}: U_{j} \rightarrow \varphi_{j}\left(U_{j}\right) \subset Y-$ диффеоморфизмы, $U \subset \bigcup_{j \in E} U_{j}$, $\operatorname{card}(E)<\aleph_{0}, 0<\varepsilon<1, l=t$ при $t<\infty, l \in \mathbb{N}$ при $t=\infty$. Используя формулу Тейлора, теорему Стоуна-Вейерштрасса и локально конечное $C^{\infty}$-разбиение единицы в многообразии $C^{\infty}(M, M)$, состоящем из $C^{\infty}$-отображений $f: M \rightarrow M$, для любого $\delta>0$ и $h \in W$ найдем в силу результатов работ [13], [14], [10] однопараметрическую подгруппу $\left\langle f^{b}: b\right\rangle$ либо с $b \in \mathbf{T}$, либо с $b \in \mathbf{L}(\mathbf{L}=\mathbb{R}$ или $\mathbf{L}$ - локальное поле), либо с $b \in B(\mathbf{L}, 0,1)$ в шаре единичного радиуса, содержащем 0 локального поля, причем $f \in W \cap G^{\prime} \cap \operatorname{Diff}{ }^{\infty}(M)$, так что $\rho_{U}^{l}(f, g)<\delta$, так как $\operatorname{Diff}^{t}(M)$ является окрестностью тождественного отображения $\mathrm{id}(x)=x$ в $C^{t}(M, M)$, где $f=f^{b_{0}}$ для соответствующего значения $b=b_{0}$. Это означает, что семейство $K$ плотно в $W$ в случае таких групп диффеоморфизмов. Для сепарабельной не локально компактной группы Банаха-Ли $G$ существование $K$ и $W$ с $K \cap W=W$ хорошо известно [15].

Поскольку $G$ сепарабельна и паракомпактна, существует локально конечное покрытие $\left\{g_{j} W_{j}: j \in \mathbb{N}, g_{j} \in G^{\prime}\right\}$ групшы $G$, где $W_{j}$ открыты и $e \in W_{j} \subset W[15],[3]$. Построим алгебраическую группу $S$ всех отображений $f$ из $\mathbb{N}$ в $H$ с поточечным умножением $\left(f_{1} f_{2}\right)(n)=f_{1}(n) f_{2}(n)$ для любых $n \in \mathbb{N}$, где $H$ - факторгруппа $F / E$ свободной полной топологической группы $F$, порожденной топологическим пространством

$$
X=\left(\prod_{j=1}^{\infty} g_{j}{ }^{\mathbf{L}}\right) \times \prod_{Y \in K} Y
$$

а $E$ - наименьшая замкнутая подгруппа в $F$, содержащая все элементы вида $g_{j}^{a+b} g_{j}^{-a} g_{j}^{-b}$, $g_{j}^{a} g_{j}^{b} g_{j}^{-a} g_{j}^{-b}, Y^{a+b} Y^{-a} Y^{-b}$ и $Y^{a} Y^{b} Y^{-a} Y^{-b}$ для любых $j \in \mathbb{N}, a, b \in \mathbf{L}$ и $Y \in K$. Это означает, что имеется вложение всех $g_{j}^{\mathbf{L}}$ и каждой однопараметрической подгруппы из $K$ в $H$. Тогда для любого $g \in \operatorname{Diff}^{t}(M)$ существует последовательность $\left\{h_{k}:=g_{j_{k}} f_{k}\right.$ : $\left.k \in \mathbb{N}, f_{k}^{b} \in K\right\} \mathrm{clim}_{k \rightarrow \infty} h_{k}=g$ (это же верно для групп Банаха-Ли $G$ ). Существование $\mu$ и $G^{\prime}$ доказано в [16], [10], [11], [17]. Тогда в случае групп Банаха-Ли и групा диффеоморфизмов существует факторное отображение $q$ соответствующей групшы $S$ на группу $G[9]$. Снабдим $S$ слабейшей топологией и соответствующей равномерностью, при которой $q$ непрерьвно и $S$ является $T_{1} \cap T_{3.5}$-пространством [3]. 
Каждая однопараметрическая подгруппа $\left\langle g^{b}: b\right\rangle$ замкнута в $G^{\prime}$ и $G$. Поэтому существует (непрерьвная) ретракция $\eta: G \rightarrow\left\langle g^{b}: b\right\rangle$ [3], т.е. $\eta \circ \eta=\eta$ и $\left.\eta\right|_{\left\langle g^{b}: b\right\rangle}=\mathrm{id} ;$ конечно, в общем случае $\eta$ необязательно является групповым гомоморфизмом. Это порождает меpy $\nu_{\left\langle g^{b}\right\rangle}(A):=\mu\left(\eta^{-1}(A)\right)$, где $A \in B f\left(\left\langle g^{b}: b\right\rangle\right)$. Благодаря квазиинвариантности $\mu$ на $G$ относительно $G^{\prime}$ существует функция $h: G \rightarrow[0, \infty)$ такая, что $\nu_{\left\langle g^{b}: b\right\rangle}(d x)=h(x) \lambda(d x)$, где $\lambda$ - мера Хаара на локально компактной группе $\left\langle g^{b}: b\right\rangle$ и $h \in L^{1}\left(\left\langle g^{b}: b\right\rangle ; \lambda, \mathbb{C}\right)[5]$. Аналогично части 1) для каждой однопараметрической подгрупшы $\left\langle g^{b}: b\right\rangle$ можно построить семейства $G_{i, j}$ компактно порожденных подгрупш $G_{C}$, где $g=g_{i} \in K, i \in \mathbb{R}$, $\nu_{\left\langle g^{b}: b\right\rangle}\left(G_{C}\right)=0$ и $G_{C_{1}} \cap G_{C_{2}}=\left\{e_{i}\right\}$ для любых $G_{C_{1}} \neq G_{C_{2}} \in G_{i, j}$. После этого построим автоморфизмы подгрупп $G_{0} \subset G$, удовлетворяющих условиям i)-iv) с $X \in K$ и $g_{j}^{\mathrm{L}}$ вместо $\mathbf{T}_{i}$. Повторяя рассуждения с леммой Куратовского-Цорна, получим автоморфизм $f \in \operatorname{Aut}(G)$ с $\beta=$ с и $G_{0}=G$ (см. также [18]). Для любых $i$ и любых $U$, открытых в $\left\langle g_{i}^{b}: b\right\rangle$, выполняется условие $f^{-1}\left(\eta^{-1}(U)\right) \in A f(G, \mu)$. Пространство $G$ имеет счетную базу топологии, а мера $\mu$ радонова и регулярна, $G^{\prime}$ плотно в $G$. Следовательно, автоморфизм $f \mu$-измерим, но разрьвен.

\section{СПИСОК ЦИТИРОВАННОЙ ЛИТЕРАТУРЫ}

[1] Bichteler K. On the existence of noncontinuous irreducible representations of a locally compact group // Invent. Math. 1968. V. 6. P. 159-162.

[2] Хьюитт Э., Росс К. А. Абстрактный гармонический анализ. М.: Наука, 1975.

[3] Энгелькинг Р. Общая топология. М.: Мир, 1986.

[4] Федерер Г. Геометричекая теория меры. М.: Наука, 1987.

[5] Fell J. M. G., Doran R.S. Representations of *-Algebras, Locally Compact Groups, and Banach *-Algebraic Bundles. Boston: Acad. Press, 1988.

[6] Dalecky Yu. L., Fomin S. V. Measures and Differential Equations in Infinite-Dimensional Space. Dordrecht, The Netherlands: Kluwer, 1991.

[7] Куратовский К. Топология. М.: Мир, 1966, 1969.

[8] Келли Дж. Общая топология. М.: Наука, 1980.

[9] Марков А.А. О свободных топологических группах // Изв. АН СССР. 1945. Т. 9. № 1. C. 3-64.

[10] Людковский С. В. Меры на группах диффеоморфизмов неархимедовых банаховых многообразий // УМН. 1996. Т. 51. № 2. С. 169-170.

[11] Людковский С.В. Меры на группах диффеоморфизмов неархимедовых многообразий, представление групп и их применения // ТМФ. 1999. Т. 119. №3. С. 381-396.

[12] Прессли Э., Сигал Г. Группы петель. М.: Мир, 1990.

[13] Araujo J., Schikhof W.H. The Weierstrass-Stone approximation theorem for $p$-adic $C^{n}$-functions // Ann. Math. Blaise Pascal. 1994. V. 1. №1. P. 61-74.

[14] Ebin D.G., Marsden J. Groups of diffeomorphisms ano the motion of an incompressible fluid // Ann. Math. (2). 1970. V. 92. P. 102-163.

[15] Бурбаки Н. Группы и алгебры Ли. М.: Мир, 1976.

[16] Далецкий Ю. Л., Шнирельман Я.И. Диффузия и квазиинвариантные меры на бесконечномерных группах // Функцион. анализ и его прилож. 1969. Т. 3. С. 156-158.

[17] Шавгулидзе Е. Т. Об одной мере, квазиинвариантной относительно действия группы диффеоморфизмов конечномерного многообразия // Докл. АН СССР. 1988. Т. 303. С. 811-814.

[18] Людковский С. В. Измеримость представлений локально компактных групп // Матем. сб. 1995. Т. 186. № 2. С. 83-92.

Институт общей физики $\mathrm{PAH}$

Поступило

E-mail : ludkovsky@ran.gpi.ru

06.10 .1998

Исправленный вариант

27.01.2000 